\title{
LATISSIMUS DORSI MUSCULOCUTANEOUS FLAP RECOSNTRUCTION AFTER NIPPLE SPARING MASTECTOMY COMPLICATION: CASE REPORT
}

Jorge V Biazus'1, Andrea Damin'1, José A Cavalheiro', Angela Zucatto', Rodrigo Cericatto1, Marcia P. Melo', Anita S. Cassiano', Murilo L. Brazan

${ }^{1}$ Hospital de Clínicas de Porto Alegre, Universidade Federal do Rio Grande do Sul - Porto Alegre (RS), Brazil.

Introduction: The latissimus dorsi (LD) myocutaneous flap has been an option for partial and total breast reconstruction. The LD muscle is supplied by thoracodorsal artery and vein. These substantial vessels branches into a network of smaller tributaries, which provide a rich blood supply both for the muscle itself and for the overlying skin. Reconstruction with the LD flap is now considered to be the primary procedure of choice for many patients for reasons of robustness and low rates of complications. The indications for autologous reconstruction are variable and include women that do not want prosthetic reconstruction, have had a prior failed prosthetic reconstruction or have had prior radiation to the breast or chest wall. Case: A 45-year-old female patient with multicentric breast carcinoma underwent nipple sparing mastectomy on the right breast with prosthesis reconstruction. The patient progressed with lower quadrants skin necrosis of the right breast. Patient had no local or systemic signs of infection and no exposure of the prosthesis. Four weeks later the first surgery, a LD myocutaneous flap was performed after necrosed area debridement with nipple preservation and rescue of the first surgery prosthesis. The prosthesis was covered by the preserved major pectoral muscle and the LD flap. Patient evolved well without complications. Conclusion: The use of musculocutaneous flaps in breast reconstructive surgery has a wide variety of applications. The LD flap is robust and reliable with a failure rate of less than $1 \%$. It can be safely utilized even in "high-risk" cases such as heavy smokers and obese patients. It is an especially useful technique for treatment of implant breast reconstruction complications. 